\title{
Motivação para a prática e não prática de exercício físico: Estudo comparativo
}

\section{Motivation to practice and not to practice physical exercise: Comparative study}

\begin{abstract}
RESUMO
O principal objectivo deste estudo consistiu em conhecer os motivos que levam as pessoas à prática e à não-prática de exercício físico. A amostra foi constituída por 185 indivíduos, 79 eram do sexo masculino e 106 do sexo feminino, com idades compreendidas entre os 12 e os 75 anos $(M=29.45$; $\mathrm{DP}=13.55)$. A motivação para a prática do exercício físico foi avaliada com base no questionário QMAD. Através do questionário IMAAD (Pereira \& Vasconcelos-Raposo, 1997), foi possível avaliar a motivação para a não-prática de exercício físico. Os resultados evidenciaram que para os nãopraticantes de exercício físico, o motivo mais importante para a prática é "fazer exercício", já para os praticantes o factor mais relevante é "estar em boa condição física". O motivo mais importante para não praticar entre os não-praticantes de exercício físico, foi "por preguiça", seguido de "falta hábitos desportivos" quer para praticantes e não-praticantes de exercício físico.
\end{abstract}

Palavras-chave: exercício físico, motivação, níveis de prática de exercício

\begin{abstract}
The main objective of this study was to know the reasons that lead people to the practice and nonpractice of physical exercise. The sample consisted of 185 individuals, 79 were male and 106 female, aged between 12 and 75 years old $(M=29.45 ; S D=13.55)$. Motivation to practice physical exercise was assessed based on the QMAD questionnaire. Through the IMAAD questionnaire (Pereira \& Vasconcelos-Raposo, 1997), it was possible to determine the motivation for not practicing physical exercise. The results showed that for non-practitioners of physical exercise, the most important reason for practicing is to "exercise". In contrast, for practitioners, the most relevant factor is "being in good physical condition". For non-participants among non-practitioners of physical exercise, the most important motive was "out of laziness", followed by "lack of sports habits" for both practitioners and non-practitioners of physical exercise.
\end{abstract}

Keywords: physical exercise, motivation, levels of exercise practice

Rute Carvalho, Diana Soares, Joana Moreira, Lia Temudo. Mestre em Psicologia do Desporto. Treinadora de Futsal UEFA B. Treinadora no Grupo Desportivo de Chaves.

Endereço para correspondência: Rute Carvalho. Escola Futsal Johnson Januário, Carrazedo de Montenegro, Portugal.

e-mail: ruteccarvalho7@gmail.com 
Actualmente a actividade física e o exercício físico têm recebido o reconhecimento pelas instituições com maiores responsabilidades na educação e promoção da saúde, nomeadamente a Organização Mundial de Saúde, American College of Sports Medicine, American Psychological Association, entre outras.

A psicologia do exercício engloba o estudo do comportamento da actividade física, incluindo as variáveis psicológicas, biológicas e ambientais que determinam a qualidade, quantidade e os padrões temporais da actividade física (Buckworth \& Dishman, 2002). Quando se escolhe uma determinada modalidade não o fazemos de forma aleatória, uma vez que por detrás dessa escolha está algo ou alguém, que consciente ou inconscientemente nos "obriga" a escolher. $\mathrm{O}$ interesse ou a simples curiosidade por uma actividade física está normalmente associado à ocupação dos tempos livres ou à saúde e bem-estar físico, psíquico e social, respondendo assim às nossas necessidades individuais e sociais (Pereira \& Vasconcelos-Raposo, 1997).

O estudo da motivação é um dos alicerces mais importantes para a compreensão das diferenças individuais na prática desportiva, dado que alguns indivíduos exibem padrões motivacionais adaptativos à medida que aplicam um determinado esforço para o sucesso, persistindo na prática desportiva, enquanto outros, nas primeiras ocasiões de insucesso, abandonam a prática em questão (Steinberg \& Maurer, 1999). A motivação intrínseca tem por base o divertimento, prazer e satisfação que o sujeito retira com a sua participação nas actividades, como acontece nas recreativas ou de tempos livres, usualmente correspondendo a actividades desafiantes (Ntoumanis, 2001). A motivação extrínseca torna-se evidente quando os indivíduos praticam uma modalidade desportiva, com vista ao reconhecimento por parte do treinador ou para obterem recompensas monetárias. A prática desportiva assume-se, assim, não como uma forma de divertimento e prazer, mas como um modo de obter recompensas ou evitar consequências negativas (Pelletier, Fortier, Vallerand, Tuson, Briere \& Blais, 1995).

Neste âmbito, existe um tipo de comportamento que se considera que o indivíduo não esteja intrínseca ou extrinsecamente motivado, sendo este designado como comportamento amotivado. Este é iniciado e regulado por "forças" para além do controlo intencional do indivíduo, tendo em conta a ausência de intenção (Deci \& Ryan, 1985).

Um dos motivos que afecta o desempenho e a participação é a motivação para a realização. Esta está relacionada com os esforços de um indivíduo em dominar uma tarefa, atingir os seus limites, superar obstáculos, e ter um melhor desempenho que os outros e ter orgulho do seu talento (Deschamps \& Domingues-Filho, 2005).

Através da análise de vários estudos, que foram efectuados no seguimento deste tema, reunimos as conclusões daqueles que nos pareceram os mais relevantes para a constituição da base teórica do nosso estudo.

Num estudo realizado com jovens portugueses verificou-se que os motivos de maior importância para a prática de actividade física eram, segundo Serpa (1992), aspectos como condição física, trabalho em equipa, aprendizagem de novas técnicas, espírito de equipa, prática de exercício, manutenção da forma, nível desportivo mais elevado, melhoria de capacidades técnicas e novas amizades (Catita, s.d.).

Num estudo orientado no sentido de perceber a motivação para a prática de natação pura desportiva, concluiu-se que a "melhoria e desenvolvimento de competências", "forma física", "atmosfera de equipa" e "desafio" foram os aspectos mais valorizados, enquanto os motivos "ser popular e conhecido", "ter a sensação de ser importante" e "pretexto para sair de casa" são os menos valorizados (Fernandes, 2004).

Com o objectivo de conhecer quais os 
factores motivacionais que conduzem à prática de voleibol, foi realizado um estudo com adolescentes, no Brasil. Os resultados apontam que o contexto competitivo mostrou ser o factor mais significativo englobando categorias em que a percentagem de resposta atingiu os $77 \%$, como a "excitação e desafios", ou $69 \%$ como a "afiliação". Ainda de encontro a este estudo, foi avaliado um constructo que, embora não incluído nas categorias motivacionais, constitui-se como um auxílio importantíssimo para a realização do questionário: O "ter alegria" teve uma concordância de resposta de $100 \%$, o que o torna um conceito de grande relevância para os profissionais de Educação Física (Paim, 2003).

Atletas juvenis desenvolvem relações de amizade muito importantes com o grupo e com o treinador (Estriga \& Cunha, 2003), pelo que este facto vai de encontro a estudos precedentes, uma vez que se apresenta como fulcral o sentimento de pertença como um motivo para a prática de actividade física. Por sua vez, foi feita a análise da motivação para a prática de desporto escolar, no concelho de Viseu, onde se retiraram as seguintes conclusões: i) os atletas orientavam-se fundamentalmente para a tarefa; ii) percepcionavam um clima motivacional essencialmente orientado para a mestria; iii) sentiam níveis relativamente elevados de prazer/interesse e esforço/ importância, não se sentindo pressionados ou tensos no decorrer da sua prática desportiva escolar; iv) apresentaram como motivos fundamentais para a sua decisão de praticarem desporto escolar os relacionados com o desenvolvimento de competências, a afiliação geral, a forma física, a competição e o prazer; v) e, de uma forma geral, declararam terem intenção de continuar a praticar desporto escolar no ano seguinte (Sousa \& Fonseca, 2004).

Os principais resultados sugerem uma prevalência de comportamentos associados a razões de causalidade interna, denotando a importância de experiências anteriores, que possam ter indiciado pensamentos negativos acerca do autoconceito e percepções de competência desportiva (Fernandes, Lázaro, \& Vasconcelos Raposo, 2004). Este estudo tem como principais objectivos identificar os motivos para a prática desportiva mais valorizados quer por homens quer por mulheres; identificar os motivos para a prática desportiva menos valorizados por cada um dos sexos; diferenciar os motivos mais valorizados, consoante diferentes níveis de envolvimento desportivo (não-praticante, praticante de EF, praticante desportivo); identificar os motivos menos valorizados em conformidade com os diferentes níveis de envolvimento desportivo (não-praticante, praticante de EF, praticante desportivo).

Com este estudo pretende-se conhecer os processos motivacionais que estão na base da prática de exercício físico e desporto, bem como os motivos pelos quais as pessoas não praticam qualquer tipo de actividade física.

\section{MÉTODOS}

O corrente estudo é de natureza transversal, já que os dados foram recolhidos num único momento temporal. Este é, ainda, exploratório e quantitativo pois é orientado para os resultados.

\section{Amostra}

A amostra utilizada neste estudo foi composta por 185 indivíduos, 106 mulheres (57.3\%) e 79 homens (42.7\%), com idades compreendidas entre os 12 e os 75 anos $(\mathrm{M}=$ 29.45; DP = 13.55). Dos inquiridos, 83 afirmaram não praticar qualquer tipo de exercício (44.9\%), 70 eram praticantes de exercício físico $(37.8 \%)$ e 32 praticavam desporto (17.3\%).

\section{Instrumentos}

Os instrumentos utilizados no presente estudo foram os questionários: Inquérito de Motivação para a Ausência de Actividade Desportiva - IMAAD (Pereira \& Vasconcelos-Raposo, 1997) e Questionário de Motivos para 
a Actividade Desportiva - QMAD é uma versão traduzida e adaptada por Serpa e Frias (1990) do Participation Motivation Questionnaire (PMQ; Gill, Gross \& Huddleston, 1983) que visam averiguar a motivação para a não prática de exercício físico e para a prática de exercício físico, respectivamente.

O primeiro questionário contém 39 itens que aquando do processo de validação se verifica, estão agrupados em 5 factores que os agrupam em "Aversão desportiva/Insatisfação" (Alpha de Cronbach de .666), "Estética/Incompetência" (Alpha de Cronbach de 0.789), "Falta de apoios/ condições" (Alpha de Cronbach de .686), "Desinteresse pelo esforço físico" (Alpha de Cronbach de .741) e "Falta de tempo" (Alpha de Cronbach de .558), apresentando todos eles um nível de consistência satisfatório, excepto este último que é medíocre. Atende a uma escala do tipo Lickert de 5 pontos ( 1 = Discordo Plenamente a $5=$ Concordo Plenamente).

O QMAD é constituído por 30 itens que foram posteriormente agrupados em 6 factores, designadamente, "Realização/Estatuto" (Alpha de Cronbach de .563), "Objectivo desportivo" (Alpha de Cronbach de .851), "Orientação para o grupo" (Alpha de Cronbach de .621), "Exercitação" (Alpha de Cronbach de .773), "Divertimento" (Alpha de Cronbach de .770) e "Influência social" (Alpha de Cronbach de .598), onde os factores 2 , 4 e 5 ostentam um nível de consistência satisfatório enquanto os restantes são medíocres, também ao nível da sua consistência. Em semelhança ao IMAAD, também o QMAD apresenta uma escala tipo Likert de 5 pontos $(1=\mathrm{b}$ Nada Importante a 5 $=$ Totalmente Importante).

À parte destes dois questionários juntámos algumas variáveis que nos pareceram relevantes para enriquecer o nosso estudo, que serviram por consequência para a recolha de dados. Desta forma, acrescentámos as variáveis sexo e idade do inquirido, bem como os níveis de prática de actividade física (Não-praticante; Praticante de Exercício Físico; Praticante de Desporto) deste, para melhor entendermos a sua posição e afinidade com a prática (ou não) de actividade física.

\section{Procedimentos}

$\mathrm{O}$ instrumento foi aplicado aleatoriamente, pelas autoras deste estudo, em várias regiões do norte de Portugal. Os inquiridos foram verbalmente informados sobre a diferença entre o exercício físico e desporto antes do início do preenchimento do questionário. Assim, definiu-se exercício físico como sendo "qualquer actividade física planeada, estruturada e repetitiva cujo objectivo é adquirir, manter ou melhorar um ou mais componentes da forma física" (Caspersen, Powell, \& Christenson, 1985); quanto ao desporto interpretámo-lo como qualquer actividade física de natureza competitiva, regida por regras institucionalizadas. Os não-praticantes foram identificados como sendo aqueles que não praticavam qualquer tipo de actividade física aquando do momento da recolha de dados, para ser considerado praticante de exercício físico teria que praticar pelo menos uma vez por semana. Foram recolhidos 200 questionários, mas 15 foram, o que representa uma mortalidade de $7.5 \%$, deixando assim uma amostra total de 185 indivíduos.

A análise estatística dos dados foi efectuada a partir da utilização do programa informático SPSS (Statitical Package for the Social Sciences) na versão 15.0, para Windows. Foi utilizada a estatística descritiva de tendência central para a descrição das variáveis (média e desvio padrão), bem como a análise da distribuição das frequências (normalidade) através do Skewness (mede a assimetria da distribuição das frequências) e do Kurtosis (mede o achatamento da distribuição), e ainda a análise da variância univariada (ANOVA) que foi utilizada com o intuito de comparar os grupos de diferentes níveis de prática desportiva e a sua significância estatística. Utilizou-se o t-test para comparar o sexo masculino e feminino quanto aos factores motivacionais para a 
prática e não prática de actividade física. Foi feito ainda o teste de Alpha de Cronbach para verificar o nível de consistência interna dos questionários utilizados.

\section{RESULTADOS}

Os dados obtidos apresentam uma distribuição normal, uma vez que os valores de
Skewness e Kurtosis se encontram entre -1 e 1. Os valores de Alpha de Cronbach, apresentam valores que oscilam entre .563 e .851. Inicialmente foram identificados cinco constructos relativamente aos motivos para a prática e outros cinco para os motivos apontadas pelas pessoas para não praticarem qualquer actividade (ver Quadro 1).

Quadro 1

Média, desvio padrão, valores de Skewness, de Kurtosis e Alpha de Cronbach dos factores do QMAD e IMAAD.

\begin{tabular}{|c|c|c|c|c|c|c|}
\hline & & $\mathbf{M}$ & DP & Skewness & Kurtosis & $\alpha$ \\
\hline \multirow{6}{*}{$\sum_{0}^{\infty}$} & “Realização estatuto” & 2.25 & .85 & .482 & -.206 & .563 \\
\hline & "Objectivos desportivos" & 3.59 & .64 & -.203 & .374 & .851 \\
\hline & "Orientação para o grupo" & 3.26 & .89 & -.032 & -.171 & .621 \\
\hline & "Exercitação" & 3.71 & .77 & -.426 & .491 & .773 \\
\hline & "Divertimento" & 3.12 & .63 & .053 & .224 & .770 \\
\hline & "Influência Social" & 2.59 & .72 & .195 & .469 & .598 \\
\hline \multirow{5}{*}{ 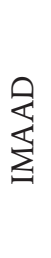 } & "Aversão desportiva/insatisfação" & 2.64 & .57 & -.055 & -.238 & .666 \\
\hline & "Estética/Incompetência" & 2.95 & .60 & -.148 & -.073 & .789 \\
\hline & "Falta de apoios/condições" & 2.64 & .62 & -.114 & -.163 & .686 \\
\hline & "Desinteresse pelo esforço físico" & 3.34 & .58 & -.098 & -.280 & .741 \\
\hline & "Falta de tempo" & 3.49 & .80 & -.722 & .053 & .558 \\
\hline
\end{tabular}

Nas comparações entre sexos, verificou-se que existe diferença, em relação ao factor "realização estatuto" ( $p=.01)$. Constatou-se que o sexo feminino atribui menos importância a este factor $(2.13 \pm 0.74)$ do que indivíduos do sexo masculino $(2.41 \pm 0.96)$. Relativamente aos factores a que o sexo feminino atribui maior importância, podemos destacar "orientação para o grupo" (3.32 \pm 0.88$)$, "aversão desportiva/ insatisfação" (2.67 \pm 0.60$)$, "estética/ incompetência" (2.97 \pm 0.61$)$ e "falta de tempo" $(3.51 \pm 0.80)$ (ver quadro 2$)$.

Quadro 2

Média e Desvio Padrão resultante da comparação entre sexos relativamente aos factores do QMAD e IMAAD.

\begin{tabular}{|c|c|c|c|c|c|}
\hline & & $\begin{array}{c}\text { Feminino } \\
M \pm \mathrm{DP}\end{array}$ & $\begin{array}{c}\text { Masculino } \\
M \pm \mathrm{DP}\end{array}$ & $t$ & $p$ \\
\hline \multirow{6}{*}{$\underset{8}{\$}$} & "Realização estatuto" & $20.13 \pm 0.74$ & $20.41 \pm 0.96$ & -2.17 & .003 \\
\hline & "Objectivos desportivos" & $30.62 \pm 0.62$ & $30.53 \pm 0.66$ & 1.03 & .30 \\
\hline & “Orientação para o grupo" & $30.32 \pm 0.88$ & $30.18 \pm 0.89$ & 1.09 & .28 \\
\hline & "Exercitação" & $30.71 \pm 0.73$ & $30.72 \pm 0.82$ & -.07 & .95 \\
\hline & "Divertimento" & $30.14 \pm 0.61$ & $30.10 \pm 0.66$ & .60 & .58 \\
\hline & "Influência Social" & $20.56 \pm 0.68$ & $20.63 \pm 0.78$ & -.70 & .52 \\
\hline \multirow{5}{*}{$\sum_{i}$} & "Aversão desportiva/insatisfação" & $20.67 \pm 0.60$ & $20.60 \pm 0.52$ & .76 & .45 \\
\hline & "Estética/Incompetência" & $20.97 \pm 0.61$ & $20.93 \pm 0.60$ & .43 & .67 \\
\hline & "Falta de apoios/condições" & $20.64 \pm 0.59$ & $20.64 \pm 0.65$ & -.03 & .98 \\
\hline & "Desinteresse pelo esforço físico" & $30.33 \pm 0.61$ & $30.34 \pm 0.54$ & -.16 & .87 \\
\hline & "Falta de tempo" & $30.51 \pm 0.80$ & $30.46 \pm 0.80$ & .48 & .64 \\
\hline
\end{tabular}


No quadro 3 apresentam-se os valores de comparação dos grupos de diferentes níveis de prática desportiva. Nesta verificou-se que apenas para 3 factores, nomeadamente "objec- tivos desportivos" ( $p=.003)$, "orientação para o grupo" $(p=.00)$ e "falta de tempo" $(p=$ $.01)$, existe uma diferença de médias significativas $(p \leq .01)$.

\section{Quadro 3}

Valores da ANOVA para os três grupos de níveis de prática de actividade física.

\begin{tabular}{|c|c|c|c|c|c|c|}
\hline & & $\begin{array}{c}\text { Não Praticante } \\
\text { E.F } \\
\text { M } \pm \text { DP }\end{array}$ & $\begin{array}{c}\text { Praticante de } \\
\text { E.F } \\
\mathrm{M} \pm \mathrm{DP} \\
\end{array}$ & $\begin{array}{c}\text { Praticante de } \\
\text { Desporto } \\
\mathrm{M} \pm \mathrm{DP} \\
\end{array}$ & $F$ & $p$ \\
\hline \multirow{6}{*}{$\sum_{0}^{9}$} & "Realização estatuto" & $2.22 \pm 0.77$ & $2.17 \pm 0.87$ & $2.51 \pm 0.98$ & 1.85 & 0.16 \\
\hline & “Objectivos desportivos” & $3.36 \pm 0.65$ & $3.68 \pm 0.54$ & $3.97 \pm 0.60$ & 13.15 & 0.00 \\
\hline & "Orientação para o grupo" & $3.11 \pm 0.81$ & $3.23 \pm 0.86$ & $3.72 \pm 1.00$ & 5.84 & 0.00 \\
\hline & “Exercitação” & $3.67 \pm 0.66$ & $3.78 \pm 0.81$ & $3.64 \pm 0.94$ & 0.56 & 0.57 \\
\hline & "Divertimento" & $3.00 \pm 0.62$ & $3.19 \pm 0.68$ & $3.12 \pm 0.63$ & 2.60 & 0.08 \\
\hline & "Influência Social" & $2.53 \pm 0.74$ & $2.67 \pm 0.75$ & $2.59 \pm 0.60$ & 0.77 & 0.47 \\
\hline \multirow{5}{*}{ 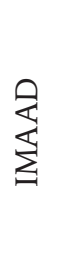 } & "Aversão desportiva/insatisfação" & $2.73 \pm 0.60$ & $2.56 \pm 0.54$ & $2.58 \pm 0.49$ & 2.00 & 0.14 \\
\hline & "Estética/Incompetência” & $3.03 \pm 0.60$ & $2.84 \pm 0.60$ & $2.98 \pm 0.61$ & 1.94 & 0.15 \\
\hline & "Falta de apoios/condições" & $2.75 \pm 0.64$ & $2.56 \pm 0.53$ & $2.51 \pm 0.66$ & 2.62 & 0.08 \\
\hline & "Desinteresse pelo esforço físico" & $3.35 \pm 0.58$ & $3.31 \pm 0.58$ & $3.35 \pm 0.60$ & 0.10 & 0.90 \\
\hline & "Falta de tempo" & $3.69 \pm 0.77$ & $3.32 \pm 0.82$ & $3.32 \pm 0.73$ & 5.21 & 0.01 \\
\hline
\end{tabular}

Seguidamente procedeu-se à identificação dos 5 itens de maior e menor concordância, relativamente aos dois questionários, nos 3 grupos.

Quadro 4

Itens de maior e menor concordância, referentemente aos dois questionários, para os não praticantes de exercício físico.

\begin{tabular}{llll}
\hline Itens do QMAD & $\mathbf{M} \pm \mathbf{D P}$ & Itens do IMAAD & M \pm DP \\
\hline Fazer exercício & $3.87 \pm 0.79$ & Por preguiça & $4.07 \pm 0.84$ \\
Manter a forma & $3.83 \pm 0.84$ & Falta de tempo & $3.97 \pm 0-92$ \\
Estar em boa condição física & $3.81 \pm 0.92$ & Falta de hábitos desportivos & $3.94 \pm 0.77$ \\
Libertar tensão & $3.73 \pm 0.77$ & Falta de interesse/vontade & $3.89 \pm 0.90$ \\
Descarregar energias & $3.61 \pm 0.71$ & O trabalho não permite & $3.70 \pm 1.03$ \\
Pretexto para sair de casa & $2.07 \pm 0.88$ & O desporto não traz benefícios & $1.78 \pm 0.99$ \\
Ser conhecido & $2.08 \pm 0.93$ & O desporto é “aborrecido, maçador e chato" & $2.07 \pm 0.97$ \\
Ter a sensação de ser importante & $2.14 \pm 0.88$ & Não querem mudar o visual & $2.12 \pm 0.93$ \\
Ser reconhecido e ter prestígio & $2.29 \pm 0.97$ & Os pais não deixam/apoiam a prática de desportiva & $2.20 \pm 0.81$ \\
Influência dos treinadores & $2.31 \pm 0.91$ & Não gostam de estar em grupo & $2.25 \pm 0.95$ \\
\hline
\end{tabular}

Relativamente ao IMAAD, o item de menor concordância, em todos os grupos, é "o desporto não traz benefícios" (não praticantes: $1.78 \pm 0.99$; praticantes de exercício físico: $1.34 \pm 0.63$; praticantes de desporto: $1.63 \pm 1.07$ ), sendo os outros quatro também semelhantes, diferindo apenas nas suas médias. Praticantes e não praticantes de exercício físico concordam que as pessoas não praticam exercício físico "por preguiça" (não praticantes: $4.07 \pm 0.84$; praticantes de exercício: $4.13 \pm 1.17)$; já para os praticantes de desporto, 
o motivo mais importante para a não prática de exercício é a "falta de hábitos desportivos" $(4.09 \pm 1.17)$.
Nos três grupos, os itens de maior concordância do QMAD são semelhantes, no entanto, as médias do grupo de não-praticantes de exer-

Quadro 5

Itens de maior e menor concordância, referentemente aos dois questionários, para os praticantes de exercício físico.

\begin{tabular}{llll}
\hline Itens do QMAD & $\mathbf{M} \pm \mathbf{D P}$ & Itens do IMAAD & M \pm DP \\
\hline Fazer exercício & $4.39 \pm 0.82$ & Por preguiça & $4.13 \pm 1.17$ \\
Estar em boa condição física & $4.34 \pm 0.82$ & Falta de interesse/vontade & $3.97 \pm 0.90$ \\
Manter a forma & $4.20 \pm 0.83$ & Falta de hábitos desportivos & $3.91 \pm 0.90$ \\
Divertimento & $4.01 \pm 0.86$ & Não gostam de se submeter ao esforço físico & $3.53 \pm 0.56$ \\
Libertar tensão & $3.87 \pm 0.87$ & Falta de tempo & $3.49 \pm 1.21$ \\
Ter a sensação de ser importante & $2.04 \pm 1.00$ & O desporto não traz benefícios & $1.34 \pm 0.63$ \\
Ser conhecido & $2.04 \pm 0.94$ & Os pais não deixam/apoiam a prática desportiva & $1.91 \pm 0.90$ \\
Pretexto para sair de casa & $2.23 \pm 1.12$ & Não querem mudar o seu visual & $1.97 \pm 0.98$ \\
Receber prémios & $2.29 \pm 1.12$ & O desporto é “aborrecido, maçador e chato” & $2.11 \pm 1.19$ \\
Ser reconhecido e ter prestígio & $2.81 \pm 1.14$ & Por vergonha dos outros & $2.26 \pm 1.01$ \\
\hline
\end{tabular}

Quadro 6

Itens de maior e menor concordância, referentemente aos dois questionários, para os praticantes de desporto.

\begin{tabular}{llll}
\hline Itens do QMAD & M \pm DP & Itens do IMAAD & M DP \\
\hline Estar em boa condição física & $4.25 \pm 0.76$ & Falta de hábitos desportivos & $4.09 \pm 1.17$ \\
Fazer exercício & $4.06 \pm 0.84$ & Falta de interesse/vontade & $4.03 \pm 0.60$ \\
Divertimento & $4.03 \pm 0.82$ & Por preguiça & $3.84 \pm 1.46$ \\
Atingir um nível desportivo mais & $4.00 \pm 1.02$ & Falta de tempo & $3.53 \pm 0.98$ \\
elevado & $3.94 \pm 0.67$ & Não gostam de se submeter ao esforço físico & $3.38 \pm 1.18$ \\
Manter a forma & $1.84 \pm 0.95$ & O desporto não traz benefícios & $1.63 \pm 1.07$ \\
Pretexto para sair de casa & $2.34 \pm 1.04$ & Não querem mudar o seu visual & $1.97 \pm 0.70$ \\
Ser conhecido & $2.41 \pm 0.98$ & O desporto é “aborrecido, maçador e chato" & $2.03 \pm 1.20$ \\
Influência dos treinadores & $2.41 \pm 1.19$ & Não gostam de estar em grupo & $2.09 \pm 0.89$ \\
Ter a sensação de ser importante & $2.47 \pm 0.80$ & Por vergonha dos outros & $2.16 \pm 0.92$ \\
\hline Influência da família e de outros amigos & &
\end{tabular}

cício físico é inferior aos restantes; o mesmo se aplica aos itens de menor concordância, pelo que não existe diferença significativa nas médias.

\section{DISCUSSÃO}

No que refere às diferenças entre sexos, aquelas que se destacaram mais foram "realização estatuto", "orientação para o grupo", "aversão desportiva/insatisfação”, "Estética/ incompetência" e "falta de tempo". Em relação ao primeiro factor, este apresenta valores mais elevados no sexo masculino, uma vez que estes atribuem maior importância ao facto de se sentirem realizados a praticarem determinada actividade física. Quanto aos restantes quatro factores acima mencionados, é no sexo feminino que se verificaram valores mais elevados, pois as raparigas sentem uma maior motivação a praticarem exercício físico se este for em grupo. 
É ainda no sexo feminino que se verifica que a falta de tempo é um dos principais motivos para a não prática de exercício físico.

Quer os não-praticantes de exercício físico, quer os praticantes de exercício e desporto, referem que os motivos mais importantes, que levam as pessoas a praticar actividade física, são "fazer exercício", "estar em boa condição física" e "manter a forma". No entanto, os não-praticantes estão de acordo que "descarregar energias" é um motivo importante para a prática de exercício físico, e, tal como os praticantes de exercício físico, mencionam ainda que "libertar tensão" leva as pessoas à prática de exercício físico. Os praticantes de desporto enfatizam que "atingir um nível desportivo mais elevado" é um motivo relevante, e, conjuntamente com os praticam exercício físico, assumem que o "divertimento" pode ser fulcral para motivar as pessoas a praticarem exercício.

Todos os grupos partilham da opinião de que o "pretexto para sair de casa", "ter a sensação de ser importante" e "ser conhecido" são motivos pouco significativos para a prática de exercício físico. "Ser reconhecido e ter prestígio" é um dos motivos menos importantes comummente referido por não-praticantes e praticantes de exercício físico, enquanto os praticantes de desporto consideram a "influência da família e de outros amigos" pouco importante. A "influência dos treinadores" é um factor pouco importante, segundo não-praticantes e praticantes de desporto. Além destes motivos, na perspectiva dos praticantes de exercício físico, "receber prémios" é pouco influenciador para a prática de exercício.

Relativamente aos motivos para a não prática de exercício físico, "por preguiça", "falta de tempo", "falta de hábitos desportivos", "falta de interesse/vontade" foram os motivos mais relevantes, de acordo com os três grupos em estudo; excepcionalmente os não-praticantes assumem que o item "o trabalho não permite" também é significativo, em detrimento dos praticantes de exercício físico e dos praticantes de desporto que enfatizam o factor "não gostam de se submeter ao esforço físico".

Os motivos menos significativos para as pessoas não praticarem exercício físico relacionam-se com o "desporto não traz benefícios", "não querem mudar o seu visual" e "o desporto é aborrecido, maçador e chato", de acordo com os três níveis de prática de actividade física estudados. Os não-praticantes e os praticantes de exercício físico assumem, conjuntamente, que os "pais não deixam/apoiam a prática desportiva" é um factor irrelevante para a não prática de exercício. Tanto os praticantes de exercício físico como os praticantes de desporto consideram que "por vergonha dos outros" não é motivo suficiente para as pessoas não praticarem actividade física, já os não-praticantes estão de acordo com os praticantes de desporto, no que toca ao item "não gostam de estar em grupo" como motivo para a não prática.

No geral, pode concluir-se que existem diferenças significativas entre os grupos. Enquanto os não praticantes de exercício físico apontam como principais factores para a não prática a "falta de tempo", "Falta de apoios/condições", "Estética/incompetência" e "Aversão desportiva/insatisfação". Os praticantes de exercício físico apontam factores relacionados com a recreação e lazer como principais motivadores para a prática de exercício físico ("Orientação para o grupo", "Exercitação" e "Divertimento"). Já os praticantes de desporto que apontam a "Realização estatuto", "Objectivos desportivos" e "Orientação para o grupo" como factores determinantes para a prática de exercício.

\section{CONCLUSÃO}

O presente estudo veio confirmar investigações anteriores realizadas no mesmo âmbito, uma vez que, segundo Serpa (1992), Rego (1995), Neves (1996) e Pereira e Vasconcelos-Raposo (1997), os motivos mais importantes para a prática de exercício físico são "estar em forma", "melhorar capacidades", "fazer amizades" e "divertimento"; os menos impor- 
tantes são "pretexto para sair de casa", "ser importante" e "ter prestígio".

Este estudo apresenta algumas limitações que podem ter enviesado ou condicionado os resultados. Alguns sujeitos responderam incorrectamente aos questionários, o que levou à eliminação dos mesmos e consequente redução da amostra. Se esta fosse maior, as margens de erro relativas à generalização dos resultados seriam menores.

Esta investigação é algo inovadora pois utiliza dois questionários em simultâneo, que contemplam o constructo da motivação (QMAD e IMAAD), e compara três grupos com diferentes níveis de prática de actividade física. Assim, a escassa literatura existente não permitiu que se fizessem muitas comparações e/ou confirmações dos resultados obtidos.

Agradecimentos:

Nada declarado.

Conflito de Interesses:

Nada declarado.

\section{Financiamento:}

Nada declarado.

\section{REFERÊNCIAS}

Buckworth, J. \& Dishman, R. (2002). Exercise psychology. Illinois: Human Kinetics.

Caspersen, C., Powell, K. \& Christenson, G. (1985). Physical activity, exercise, and physical fitness: definitions and distinctions for health-related research. Public Health Reports, 100 (2), 126-128.

Catita, L. (2002). A importância do treinador na motivação e continuidade da prática de jovens atletas em luta. Luta Magazine, 1.

Cid, F. (2002, Dezembro). Alteração dos motivos para a prática desportiva em crianças e jovens. Lecturas, Educación Física y Deportes, Revista Digital. https://www. efdeportes.com/efd55/motiv1.htm

Deschamps, S. \& Domingues-Filho, L. (2005). Motivos e benefícios psicológicos que levam os indivíduos dos sexos masculino e feminino a praticarem ciclismo indoor. Revista Brasileira Ciência e Movimento, 13 (2), 27-32

Estriga, M. \& Cunha, A. (2003). Motivação para a prática do andebol: Motivos de escolha e de prática do andebol por jovens atletas do sexo feminino. Tese de Mestrado não publicada, Porto: FCDEF-UP.

Fernandes, H., Lázaro, J. \& Vasconcelos Raposo, J. (2004). Características psicométricas do inquérito de motivações para a ausência de actividade desportiva em adultos. Revista Portuguesa de Ciências do Desporto, 4(2), 143.

Fernandes, R. (2004). Motivação das nadadoras para a prática de natação pura desportiva. Revista Portuguesa de Ciências do Desporto, 4(2), 144-145.

Gill, D., Gross, J. \& Huddleston, S. (1983). Participation motivation in youth sports. International Journal Sport Psychology,14, $1-14$.

Ntoumanis, N. (2001). A self-determination approach to the understanding of motivation in physical education. British Journal of Educational Psychology, 71, 225-242.

Paim, M. (2003, Junho). Voleibol, que fatores motivacionais levam à sua prática? Lecturas, Educación Física y Deportes, Revista Digital. 61. https://dialnet.unirioja.es/ servlet/articulo? codigo $=625130$

Pelletier, L., Fortier, M., Vallerand, R., Tuson, K., Briere, N., \& Blais, M. (1995). Toward a new measure of intrinsic motivation, extrinsic motivation, and amotivation in sports: the Sport Motivation Scale (SMS). Journal of Sport \& Exercise Psychology (JSEP), 17(1), 35 - 53. doi: 10.1123/jsep.17.1.35

Pereira, J. \& Vasconcelos Raposo, J. (1997). As motivações e a prática desportiva: Análise 
descritiva, factorial e comparativa dos motivos e factores motivacionais para a participação e não-participação desportiva de jovens do Documento Não publicado. Porto: FCDEF-UP

Ryan, R. \& Deci, E. (2000). Self-Determination theory and the facilitation of intrinsic motivation, social development and wellbeing. American Psychologist, 55, 68-78.

Serpa, S. \& Frias, J. (1990). Estudo da relação professor/aluno em ginástica de representação e manutenção. Dissertação de licenciatura não publicada, Lisboa: Faculdade de Motricidade Humana.

Sousa, M. \& Fonseca, A. (2004). A motivação dos jovens para a prática de desporto escolar. Estudo desenvolvido no centro de área educativa de Viseu. Revista Portuguesa de Ciências do Desporto, 4(2), 144.
Silva, M., Figueiredo, A. \& Gonçalves, C. (s.d.). Motivos para a participação desportiva: conceitos e instrumentos. Projecto PRONTALSPORT, Coimbra: Faculdade de Ciências do Desporto e Educação Física, Universidade de Coimbra.

Steinberg, G.M. \& Maurer, M. (1999). Multiple goal strategy: Theoretical implications and pratical approaches for motor skill instruction. Journal of Physical Education, Recreation and Dance, 70(2). 61-65. doi: 10.1080/07303084.1999.10605667 